\title{
OSCILLATION IN SECOND ORDER FUNCTIONAL EQUATIONS WITH DEVIATING ARGUMENTS
}

\author{
BHAGAT SINGH \\ Department of Mathematics \\ University of Wisconsin Center \\ Manitowoc, Wisconsin 54220 U.S.A. \\ (Received October 16, 1979)
}

ABSTRACT. For the pair of functional equations

(A)

$$
\left(r(t) y^{\prime}(t)\right)^{\prime}+p(t) h(h(g(t)))=f(t)
$$

and

(B)

$$
\left(r(t) y^{\prime}(t)\right)^{\prime}-p(t) h(y(g(t)))=0
$$

sufficient conditions have been found to cause all solutions of equation (A) to be oscillatory. These conditions depend upon a positive solution of equation (B). KEY WORDS AND PHRASES: Oscillatory, Nonoscillatory, Sublinear, Superlinear

1980 MATHEMATICS SUBJECT CLASSIFICATION CODES:

\section{INTRODUCTION.}

Our main goal, in this work, is to seek the oscillatory behavior of the equation 


$$
\left(r(t) y^{\prime}(t)\right)^{\prime}+p(t) h(y(g(t)))=f(t),
$$

via the nonoscillation of the equation

$$
\left(r(t) y^{\prime}(t)\right)^{\prime}-p(t) h(y(g(t)))=0 .
$$

Oscillation properties of equation (1.1) were studied by Kartsatos [3] and Kusano and Onose [4] by first "homogenizing" it and then using the techniques known for homogeneous equations. In fact a function $\lambda(t)$ was sought to satisfy

$$
\left(r(t)(y(t)-\lambda(t))^{\prime}=f(t) .\right.
$$

A similar approach was later used by this author [9] in finding conditions for the oscillation of the equation

$$
\left(r(t) y^{\prime}(t)\right)^{(n-1)}+(-1)^{n+1} p(t) y(g(t))=f(t)
$$

Recently Rankin [8] presented a new approach to study the oscillatory behavior of the ordinary differential equation

$$
y^{\prime \prime}(t)+p(t) y(t)=f(t)
$$

by using the transformation

$$
y(t)=\phi(t) z(t),
$$

where $\phi(t)$ is a positive solution of the equation

$$
y^{\prime \prime}(t)+p(t) y(t)=0 \text {. }
$$

Transformations usually do not carry over to functional equations (1.1) and (1.2). The failure in study of equation (1.1) leads us to this work in which we present a different approach to study the oscillation of equation (1.1) which may be sublinear, superlinear, retarded or advanced.

Since our results do not depend on the integral size of $p(t)$, they are different from those of Kartsatos [3], Kusano and onose [4] and this author [9]. Our results are also different than those of Rankin [8]. In fact the following example shows that Rankin's results are not true for the pair of retarded equations 


$$
\begin{aligned}
& y^{\prime \prime}(t)+\frac{3}{16 t^{2}(t-\pi)^{3 / 4}} y(t-\pi)=-100 t \sin 10 t+20 \cos 10 t \\
& +\frac{3}{8}{\frac{(t-\pi)^{1 / 4}}{t^{2}}}^{1 / \frac{3(t-\pi)^{1 / 4}}{16 t^{2}}} \sin 10 t
\end{aligned}
$$

and

$$
y^{\prime \prime}(t)+\frac{3}{16 t^{2}(t-\pi)^{3 / 4}} y(t-\pi)=0
$$

Equation (1.9) has the nonoscillatory solution $\phi(t)=t^{3 / 4}$ which satisfies the conclusion of Rankin's main theorem ([8, Theorem 2]) namely

$$
\begin{aligned}
& \underset{t \rightarrow \infty}{\lim \inf } \int_{T}^{t} \frac{1}{\phi^{2}(x)} \int_{T}^{x} \phi(s) f(s) d s d x=-\infty, \\
& \underset{t \rightarrow \infty}{\lim \sup } \int_{T}^{t} \frac{1}{\phi^{2}(x)} \int_{T}^{x} \phi(s) f(s) d s d x=\infty,
\end{aligned}
$$

and $\quad \int_{T}^{\infty} \frac{1}{\phi^{2}(x)} d x<\infty$,

for any large $\mathrm{T}>0$; where

$$
f(t)=-100 t \sin (10 t)+20 \cos (10 t)+\frac{3(t-\pi)^{1 / 4}}{8 t^{2}}+\frac{3(t-\pi)^{1 / 4}}{16 t^{2}} \sin (10 t) \text {. }
$$

But equation (1.8) has the nonoscillatory solution

$$
y(t)=2 t+t \sin (10 t)
$$

\section{DEFINITIONS AND ASSUMPTIONS}

Throughout this study we assume the following:

(i) $g(t), r(t), p(t), h(t)$ and $f(t)$ are $c[R, R]$ where $R$ denotes the real line;

(ii) $\quad r(t)>0, r^{\prime}(t) \leq 0$ and $p(t)>0$ for $t>t_{0}>0$ where we shall assume $t_{0}$ to be fixed arbitrarily. $t_{0}$ will be referred to in this study without any further mention; 


$$
\begin{aligned}
& \text { (iii) } g(t) \rightarrow \infty \text { as } t \rightarrow \infty \text {; } \\
& \text { (iv) } \operatorname{sign} h(t)=\operatorname{sign} t .
\end{aligned}
$$

The term "solution" refers to nontrivial continuously extendable solutions of equations under consideration over the interval $\left[t_{0}, \infty\right)$. We call a function $Q(t) \varepsilon C\left[\left[t_{0}, \infty\right), R\right] \quad$ oscillatory if $Q(t)$ has arbitrarily large zeros on $\left[t_{0}, \infty\right)$; otherwise $Q(t)$ is called nonoscillatory. Equations (1.1) and (1.2) are called sublinear or superlinear

$$
0<\frac{h(t)}{t^{\alpha}} \leq k
$$

if $0<\alpha \leq 1$ or $\alpha<1$ respectively where $k$ is constant and $\alpha$ is the ratio of odd integers.

\section{MAIN RESULTS}

THEOREM 1: In addition to (i)-(iv) suppose there exists a function $\phi(t)$ which is continuous for $t \geq t_{0}$ and satisfies $\left(r(t) \phi^{\prime}(t)\right)^{\prime} \geq 0$ (邦 in any interval),

$$
\begin{aligned}
& \underset{t \rightarrow \infty}{\lim \inf } \int^{t} \frac{1}{\phi^{2}(s)} \int^{s} \phi(x) f(x) d x d s=-\infty, \\
& \underset{t \rightarrow \infty}{\lim \sup } \int^{t} \frac{1}{\phi^{2}(s)} \int^{s} \phi(x) f(x) d x d s=\infty
\end{aligned}
$$

and

$$
\int^{\infty} \frac{1}{\phi^{2}(t)} d t<\infty .
$$

Then all solutions of equation (1.1) are oscillatory.

PROOF: Suppose to the contrary that equation (1.1) has a nonoscillatory solution $y(t)$. Without any loss of generality suppose $T>t_{0}$ is large enough so that for $t>T, y(g(t))>0$ and $y(t)>0$. Rewriting equation (1.1) after multiplication with $\phi(t)$ we have

$$
\left(r(t) \phi(t) y^{\prime}(t)\right)^{\prime}-\left(r(t) \phi^{\prime}(t)\right) y^{\prime}(t)+p(t) \phi(t) h(y(g(t)))=\phi(t) f(t) \text {. (3.4) }
$$


Integrating (3.4) for $t \geq T$ we have

$$
\begin{aligned}
& r(t) \phi(t) y^{\prime}(t)-r(T) \phi(T) y^{\prime}(T)-r(t) \phi^{\prime}(t) y(t) \\
& +r(T) \phi^{\prime}(T) y(T)+\int_{T}^{t}\left(r(s) \phi^{\prime}(s)\right)^{\prime} y(s) d s \\
& +\int_{T}^{t} p(s) \phi(s) h(y(g(s))) d s=\int_{T}^{t} \phi(s) f(s) d s .
\end{aligned}
$$

Set

$$
K=r(T) \phi^{\prime}(T) Y(T)-r(T) \phi(T) Y^{\prime}(T)
$$

Dividing (3.5) by $\phi^{2}(t)$ and rearranging terms we have

$$
\begin{aligned}
& \frac{r(t) y^{\prime}(t)}{\phi(t)}+\frac{K}{\phi^{2}(t)}-\frac{r(t) \phi^{\prime}(t) y(t)}{\phi^{2}(t)}+\frac{1}{\phi^{2}(t)} \int_{T}^{t}\left(r(s) \phi^{\prime}(s)\right) \text { 'yds } \\
& +\frac{1}{\phi^{2}(t)} \int_{T}^{t} p(s) \phi(s) h(y(g(s))) d s=\frac{1}{\phi^{2}(t)} \int_{T}^{t} \phi(s) f(s) d s .
\end{aligned}
$$

Integrating (3.7) between $T$ and $t$ we have

$$
\begin{aligned}
& \frac{r(t) y(t)}{\phi(t)}-\frac{r(T) y(T)}{\phi(T)}+\int_{T}^{t} \frac{r(s) \phi^{\prime}(s) y(s)}{\phi^{2}(s)} d s-\int_{T}^{t} \frac{r^{\prime}(s) y(s)}{\phi(s)} d s \\
& +\int_{T}^{t} K / \phi^{2}(s) d s-\int_{T}^{t} \frac{r(s) \phi^{\prime}(s) y(s)}{\phi^{2}(s)} d s \\
& +\int_{T}^{t} \frac{1}{\phi^{2}(x)} \int_{T}^{x}\left[\left(r(s) \phi^{\prime}(s)\right)^{\prime} y(s)+p(s) \phi(s) h(y(g(s)))\right] d s d x \\
& =\int_{T}^{t} \frac{1}{\phi^{2}(x)} \int_{T}^{x} \phi(s) f(s) d s d x
\end{aligned}
$$

which leads to

$$
\frac{r(t) y(t)}{\phi(t)}-\frac{r(T) y(T)}{\phi(T)}-\int_{T}^{t} \frac{r^{\prime}(s) y(s)}{\phi(s)} d s
$$




$$
\begin{aligned}
& +\mathrm{K} \int_{T}^{\mathrm{t}} 1 / \phi^{2}(s) d s \\
& +\int_{T}^{t} \frac{1}{\phi^{2}(x)} \int_{T}^{x}\left[\left(r(s) \phi^{\prime}(s)\right) \cdot y(s)+p(s) \phi(s) h(y(g(s)))\right] d s d x \\
& =\int_{T}^{t} \frac{1}{\phi^{2}(x)} \int_{T}^{x} \phi(s) f(s) d s d x .
\end{aligned}
$$

Since third, fourth and fifth terms on the left hand side of (3.9) are either nonnegative or finite, we immediately reach a contradiction in view of (3.1) and (3.2). The proof is complete.

COROLLARY 1. Suppose (i)-(iv) hold. Further suppose that equation a positive solution $\phi(t)$ satisfying (3.1), (3.2) and (3.3). Then all solutions of equation (1.1) are oscillatory.

PROOF. Since $\left(r(t) \phi^{\prime}(t)\right)^{\prime} \geq 0$, conclusion follows from Theorem 1 .

EXAMPLE 1. Consider the equations

$$
y^{\prime \prime}(t)+e^{\pi} y(t-\pi)=4 e^{2 t} \cos t+3 e^{2 t} \sin t-e^{2 t-\pi} \sin t,
$$

and

$$
\mathrm{y}^{\prime \prime}(\mathrm{t})-\mathrm{e}^{\pi} \mathrm{Y}(\mathrm{t}-\pi)=0,
$$

for $t>\pi$. Equation (3.11) has $y(t)=e^{t}$ as a solution which satisfies (3.1), (3.2) and (3.3). Thus all solutions of equation (3.10) are oscillatory. In fact $y(t)=e^{2 t}$ sint is one such solution.

REMARK. In Rankin's work $\phi^{\prime \prime}(t)<0$ where as here $\phi^{\prime \prime}(t)>0$ when $r(t) \equiv 1$.

THEOREM 2. Suppose $r(t) \equiv 1$ and (i)-(iv) hold. Further suppose that equation (1.2) has a positive solution $\phi(t)$ such that $\phi^{\prime}(t) \geq 0$ ( subinterval) for $t>t_{0}$. Let (3.1) and (3.2) of Theorem (1) hold. Then all solutions of equation (1.1) are oscillatory. 
PROOF: Since

$\phi^{\prime \prime}(t) \geq 0, \phi^{\prime}(t) \geq 0$ and $\phi(t) \geq 0$,

for $t>t_{0}$, there exist positive numbers $c_{1}$ and $c_{2}$ such that $\phi(t) \geq c_{1} t+c_{2}$, and consequently $\phi(t)$ satisfies (3.3). The proof is complete. We now have the following corollary.

COROLLARY 2: Suppose equation (1.2) has a positive nonoscillatory solution $z(t)$ such that $z^{\prime}(t)>0$. Further suppose that equation (1.1) has a nonoscillatory solution. Then either

$$
\underset{t \rightarrow \infty}{\lim \inf } \int^{t} \frac{1}{z^{2}(s)} \int^{s} z(x) f(x) d x d s>-\infty
$$

or

$$
\underset{t \rightarrow \infty}{\lim \sup _{t \rightarrow \infty}} \int^{\infty} \frac{1}{z^{2}(s)} \int^{s} z(x) f(x) d x d s<\infty .
$$

EXAMPLE 2. The equation

$$
y^{\prime \prime}(t)+\frac{2}{t^{2}} y(t)=-\sin t+\frac{4}{t^{2}}+\frac{2 \sin t}{t^{2}} \text {. }
$$

has the nonoscillatory solution $y(t)=2+\sin t$. Now consider

$$
y^{\prime \prime}(t)-\frac{2}{t^{2}} y(t)=0 \text {, }
$$

which has $z(t)=t^{2}$ as a nonoscillatory solutions satisfying the conditions and conclusion of corollary 2 .

\section{ASYMPTOTIC NONOSCILLATION}

Example 2 shows that when (3.1) and (3.2) are relaxed then equation (1.1) may have nonoscillatory solutions. In this section we give conditions when nonoscillatory solutions of (1.1) approach limits.

THEOREM 3: Suppose (i)-(iv) hold. Let $\phi(t)$ be a positive solution of

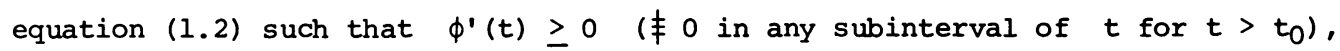




$$
\lim _{t \rightarrow \infty} \inf \int^{t} \frac{1}{\phi^{2}(x)} \int^{x} \phi(s) f(s) d s d x<0,
$$

and

$$
\underset{t \rightarrow \infty}{\lim \sup } \int^{t} \frac{1}{\phi^{2}(x)} \int^{x} \phi(s) f(s) d s d x>0 .
$$

Let $y(t)$ be a bounded solution of equation (1.1). If $y(t)$ is nonoscillatory then $y(t)$ tends to a finite limit.

PROOF. Without any loss of generality, let $T \geq t_{0}$ be large enough so that $y(t)>0$ and $y(g(t))>0$ for $t \geq T$. Suppose to the contrary that

$$
\lim _{t \rightarrow \infty} \inf y(t)<\underset{t \rightarrow \infty}{\lim \sup y(t) .}
$$

Then there exists a sequence $\left\{T_{n}\right\}_{n=1}^{\infty}$ such that $T_{n} \rightarrow \infty$ as $n \rightarrow \infty$ and $\mathrm{y}^{\prime}\left(\mathrm{T}_{\mathrm{n}}\right)=0$. Let $\mathrm{k}$ be a large positive integer such that

$$
\frac{y\left(T_{k}\right) r\left(T_{k}\right)}{\phi\left(T_{k}\right)}<\operatorname{Min}\left[-\underset{t \rightarrow \infty}{-\lim \inf } \int_{T_{k}}^{t} \frac{1}{\phi^{2}(x)} \int_{T_{k}}^{x} \phi(s) f(s) d s d x\right. \text {, }
$$

$$
\left.\underset{t \rightarrow \infty}{\lim \sup _{T_{k}}} \int_{\phi^{2}(x)}^{t} \frac{1}{T_{T_{k}}} \int^{x} \phi(s) f(s) d s d x\right] \text {. }
$$

Following the proof of Theorem 1, we obtain from (3.9)

$$
\begin{aligned}
\frac{r(t) y(t)}{\phi(t)} & -\int_{T_{k}}^{t} \frac{r^{\prime}(s) y(s) d s}{\phi(s)}+r\left(T_{k}\right) \phi^{\prime}\left(T_{k}\right) y\left(T_{k}\right) \int_{T_{k}}^{t} \frac{1}{\phi^{2}(x)} d x \\
& +\int_{T_{k}}^{t} \frac{1}{\phi^{2}(x)} \int_{T_{k}}^{x} p(s)(y(s) h(\phi(g(s)))+\phi(s) h(y(g(s)))) d s d x \\
& =\int_{T_{k}}^{t} \frac{1}{\phi^{2}(x)} \int_{T_{k}}^{x} \phi(s) f(s) d s d x+\frac{y\left(T_{k}\right) r\left(T_{k}\right)}{\phi\left(T_{k}\right)} .
\end{aligned}
$$


In view of $(4.1),(4.2)$ and $(4.4)$, we reach a contradiction in (4.5). The proof is complete.

REMARK. Example 2 shows that conditions (4.1) and (4.2) cannot be weakened.

COROLLARY 3. Suppose conditions of Theorem 3 hold. Let $y(t)$ be any solution of equation (1.1) such that $\frac{y(t)}{\phi(t)} \rightarrow 0$ as $t \rightarrow \infty$. If $y(t)$ is nonoscillatory then $y(t)$ tends to a finite or infinite limit as $t \rightarrow \infty$.

REMARK. Recently Graef and Spikes [1], Hammett [2], Kusano and Onose [5,6], Philos and Starkos [7], this author $[10,11]$ have studied asymptotic nonoscillation with regard to equation (1.1). However all these results make use of an integral condition on $p(t)$. Theorem 3 and Corollary 3 present a different approach.

\section{REFERENCES}

1. J. Graef and P. Spikes, Asymptotic behavior of solutions of a second order nonlinear differential equation, J. Differential Equations, 17 (1975), 461-476.

2. M. Hammett, Nonoscillation properties of a nonlinear differential equation, Proc. Amer. Math. Soc., 30 (1971), 92-96.

3. A.G. Kartsatos, on the maintenance of oscillations of nth order equations under the effect of a small forcing term, J. Differential Equations, 10 (1971), 355-563.

4. T. Kusano and H. Onose, Oscillations of functional differential equations with retarded arguments, 15 (1974), 269-277.

5. T. Kusano and H. Onose, Asymptotic behavior of nonoscillatory solutions of functional differential equations of arbitrary order, J. London Math. Soc., 14 (1976), 106-112.

6. T. Kusano and H. Onose, Nonoscillation theorems for differential equations with deviating argument, Pacific J. Math.? 63 (1976), 185-192.

7. Ch. G. Philos and V.A. Staikos, Asymptotic properties of nonoscillatory solutions of differential equations with deviating argument, Pacific J. Math., 70 (1977), 221-242.

8. S. Rankin, Oscillation results for a nonhomogeneous equations, Pacific J. Math., 80 (1979), 237-244. 
9. B. Singh, Impact of delays on oscillation in general functional equations, Hiroshima Math. J., 5 (1975), 351-361.

10. B. Singh, Forced nonoscillations in fourth order functional equations, Funk. Ekva., 19 (1976), 227-237.

11. B. Singh, Nonoscillations of forced fourth order retarded equations, SIAM J. Appl. Math., 28 (1975), 265-269.

12. B. Singh and T. Kusano, On asymptotic limits of nonoscillations in functional equations with retarded arguments, Hiroshima Math. J., (to appear). 


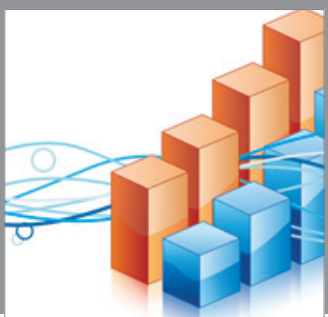

Advances in

Operations Research

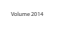

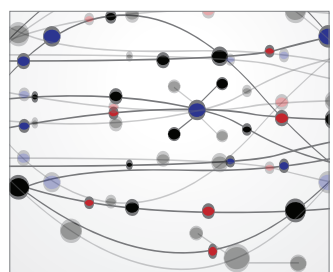

\section{The Scientific} World Journal
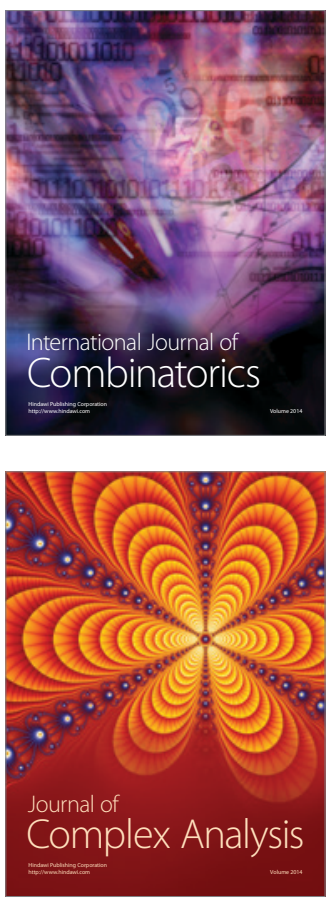

International Journal of

Mathematics and

Mathematical

Sciences
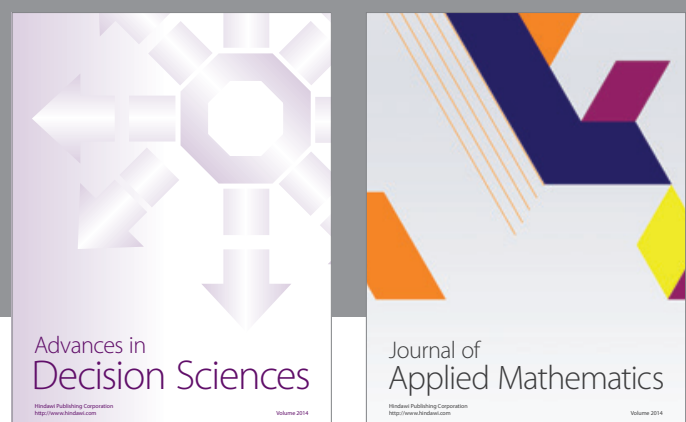

Journal of

Applied Mathematics
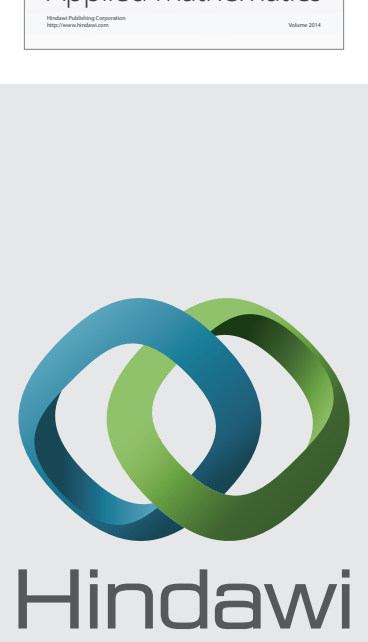

Submit your manuscripts at http://www.hindawi.com
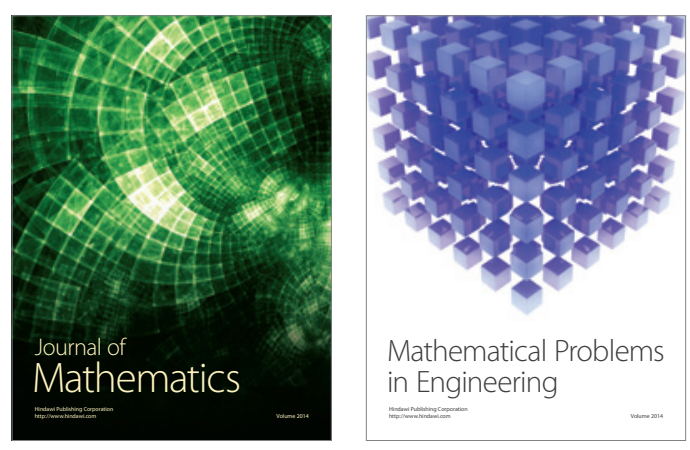

Mathematical Problems in Engineering
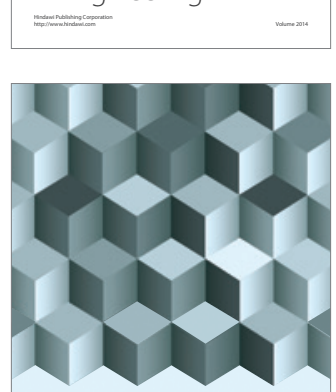

Journal of

Function Spaces
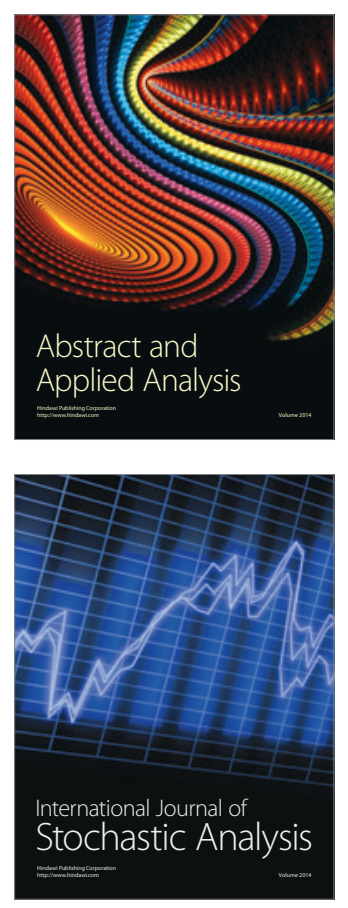

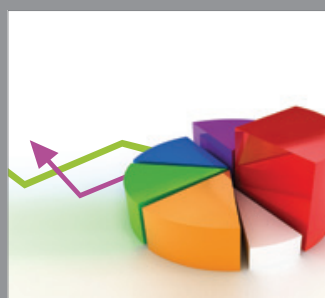

ournal of

Probability and Statistics

Promensencen
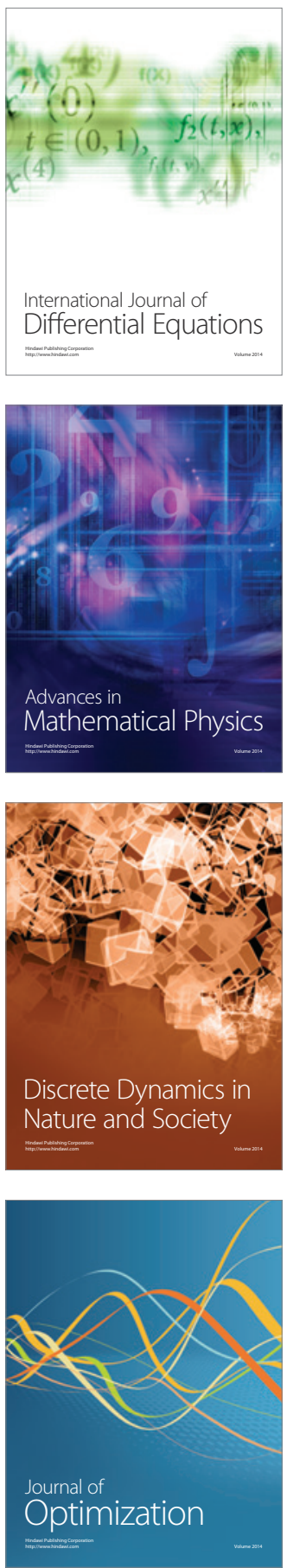